\title{
Precise Attitude Control System Design for the Tracking of Parabolic Satellite Antenna
}

\author{
Myeongkyun Kim, Jinsoo Kim and Oh Yang \\ Electronic Engineering of Cheongju University \\ Kimmk15@gmail.com,jinsoa@gmail.com,ohyang@cju.ac.kr
}

\begin{abstract}
This paper parabolic satellite antenna aims to track the precise location of a moving ship or, in the car that can be used to design the system. That was previously used with the reaction rate of a stepping motor, BLDC motor, in order to suppress the noise two-axis control and a separate encoder to the six degrees of freedom motion system was implemented in a precise location. In order to overcome various disturbances gyro sensor Yaw, Pitch, Roll 3 axes were used. To respond immediately to the disturbance at the same time, two-axis motor by controlling the precise location of the system was designed to compensate instantaneously. In addition, the circle tracking algorithm has applied for our system. We designed that the AGC signal of digital tuner quickly had gone to up to the maximum point. In order to solve the various communication errors such as moving the antenna or ground, we had designed the control system
\end{abstract}

Keywords: Parabolic Satellite Antenna Tracking System, Digital Tuner, BLDC Motor, Gyro Sensor

\section{Introduction}

Depending on the speed of faster industry information, by using satellite, broadcasting, telecommunications, high-speed Internet and other services are being made diversification. In addition, based on the domestic technology, the technology raising the satellite into orbit has been evolved. The satellite communication system using satellite in accordance with the advancement of technology and the rapidly developing, is increasing to use as international communication in many countries now as well as the domestic communication. The Soviet Union, including the United States, Canada, Japan, France and other developed countries retain their own satellite performing competitive services, and in addition many countries, including developing countries are planning to hold the satellite, or local satellite, Intelsat satellite has been used in their communication.[1-2]

As a result, in particular, recently small satellite broadcast receivers antennas that can receive satellite broadcast as high directivity are increasing significantly in demand. These days, especially the satellite to watch in the house as well as in cars, yachts, trains and moving objects equipped with a satellite system receiving services are increasing. As a result, the needed of a system from moving objects to fixing one place is increasing as time goes. As an example of a dynamic situation typically, the ship will do the six degrees of freedom motion (surge, sway, heave, roll, pitch, yaw) by the waves, wind and other meteorological changes [3].

In general, in order to capture the six degrees of freedom movement, the various sensors are needed. This paper tries to design a position control system using gyro sensor among a lot of sensors. In the case of the acceleration sensor used to position or speed control, it 
represents the increase or decrease of the rate on fixed straight line direction. However, since moving objects another that performing six degrees of freedom motion print out the sum of the two outputs, a dynamic acceleration and static acceleration, otherwise action has minimal or non-stationary state, a difficult situation occurs to measure the acceleration in one way direction with the acceleration sensor [4].

According to the movement of an object with gyro sensor, it must be able to respond immediately. As using the data obtained from gyro sensor by controlling the BLDC motor, the precise attitude control system is completed. BLDC motor detects the direction of magnetic field of rotor, that is to say, the absolute position of the rotor in general and determines the direction of the current of the stator [5-6].

This information can be identified by the output of the encoder that is attached to the rotor axis of rotation with the rotor or irritation to the output of the sensor, Hall sensor. However hall sensor is used to control the speed of BLDC motor, to control the precision position, it cannot be used due to many angle errors. The characteristic output of the encoder will be able to know the absolute position of the rotor and using the identified information by sending the appropriate current flowing to stator, it will be able to know the exact location.

This paper is in response to the six degrees of freedom motion moving rapidly and to control the exact position of satellite antenna, the three-axis Yaw, Pitch, Roll Gyro sensor mounted in the direction based on the exact location of the AGC signal analysis and digital tuner, Tuner Lock signal to the satellite estimates, BLDC motor using a two-axis control and encoder for precise position control, precise positioning design the Parabolic satellite antenna tracking system. The tracking algorithm is proposed for DBS reception and confirm the superiority by experiments.

\section{BLDC Motor Position Control}

\subsection{BLDC Motor Drive Principle}

In order to control the position of the antenna, the motor is used as required. Motors typically AC, DC, BLDC (Brushless Direct Current) motor can be divided into. BLDC motors and other motors of the biggest difference between the two different VCC and GND signals of motor behavior, but in the case of BLDC motor, the only action we need to be entered three-phase. The BLDC motor has been applied widely car industry, aerospace industry, medical equipment, factory automation, laboratory exercises as well as general office equipment, household appliances. As its name implies, BLDC motors do not use brushes for current control circuit rectified by the electronic [7].

BLDC motors have high torque characteristics for high-speed dynamic response, high efficiency, long operating life, low noise, higher speed so that there are many advantages of those field. But the price is expensive compared to other motors and the large motors have not been developed, failure to repair are the difficulties pointed out. BLDC motors do not have the same mechanical structure with brushes and slip ring contacts. This is the motor to expect in keeping with DC motors such as high-speed responsiveness, control, efficiency. BLDC motor can be expressed by dividing the part as the stator windings of each phase winding resistance losses in the current flow and the magnetic field formed by the inductor portion, representing the electrical output of the motor back electromotive force. Based on Figure 1 BLDC motor phase voltage equations can be expressed as the equation (1). 


$$
\begin{aligned}
& \mathrm{V}_{\mathrm{a}}=\mathrm{R}_{\mathrm{s}} \mathrm{i}_{\mathrm{a}}+\mathrm{L}_{\mathrm{s}} \frac{\mathrm{d}}{\mathrm{dt}} \mathrm{i}_{\mathrm{a}}+\mathrm{e}_{\mathrm{a}} \\
& \mathrm{V}_{\mathrm{b}}=\mathrm{R}_{\mathrm{s}} \mathrm{i}_{\mathrm{b}}+\mathrm{L}_{\mathrm{s}} \frac{\mathrm{d}}{\mathrm{dt}} \mathrm{i}_{\mathrm{b}}+\mathrm{e}_{\mathrm{b}} \\
& \mathrm{V}_{\mathrm{c}}=\mathrm{R}_{\mathrm{s}} \mathrm{i}_{\mathrm{c}}+\mathrm{L}_{\mathrm{s}} \frac{\mathrm{d}}{\mathrm{dt}} \mathrm{i}_{\mathrm{c}}+\mathrm{e}_{\mathrm{c}}
\end{aligned}
$$

In Equation (1), Rs indicates the value of the stator windings of each phase and Ls indicates the value of the inductance. In addition, ea, eb, ec are indicated by the value of the counter electromotive force of the stator winding, Va, Vb, Vc show the motor phase voltage, ia, ib, ic show phase current of the motor.

If the voltage put in the motor, the current flows in the stator windings of the motor and electrical output is generated. In fact, the electrical output of the motor can be movable output for the rotor. As these electrical output and unemployment mechanical output torque of the motor can be expressed as the equation (2).

$$
\mathrm{T}_{\mathrm{e}}=\frac{\mathrm{e}_{\mathrm{a}} \mathrm{i}_{\mathrm{a}}+\mathrm{e}_{\mathrm{b}} \mathrm{i}_{\mathrm{b}}+\mathrm{e}_{\mathrm{c}} \mathrm{i}_{\mathrm{c}}}{\omega_{\mathrm{m}}}
$$

Equation (2) represents the angular velocity of the motor in the $\omega_{\mathrm{m}}$. As shown in Table 1 for each of the $120^{\circ}$ phase conduction angle is presented. Figure 1 is the base system for driving the three phase BLDC motor. Circuit configuration is also configured as Figure 1.

Table 1. BLDC Motor State

\begin{tabular}{ccccccc}
\hline Mode & 1th & 2th & 3th & 4th & 5th & 6th \\
\hline \hline Hall A & 1 & 0 & 1 & 0 & 1 & 0 \\
Hall B & 0 & 1 & 1 & 0 & 0 & 1 \\
Hall C & 0 & 0 & 0 & 1 & 1 & 1 \\
S1(a+) & 1 & 0 & 0 & 0 & 1 & 0 \\
S2(b+) & 0 & 1 & 0 & 0 & 0 & 1 \\
S3(c+) & 0 & 0 & 1 & 1 & 0 & 0 \\
S4(a-) & 0 & 1 & 1 & 0 & 0 & 0 \\
S5(b-) & 0 & 0 & 0 & 1 & 1 & 0 \\
S6(Wc-) & 2 & 0 & 0 & 0 & 0 & 1 \\
\hline
\end{tabular}

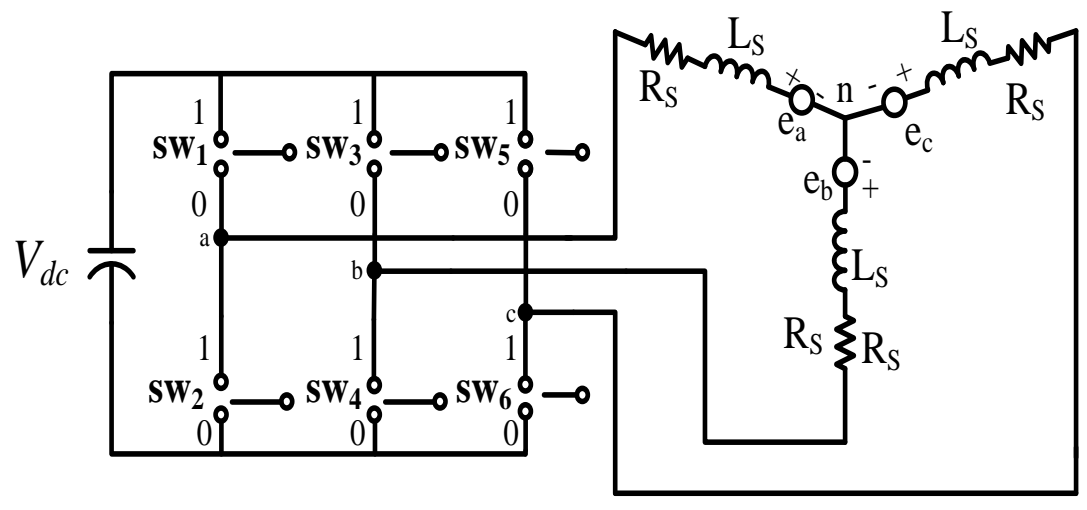

Figure 1. The Driving System of Three Phase BLDC Motor 


\subsection{PI Controller Design for BLDC Motor Position Control}

It is indicated a control technique by integrating the error signal to produce a control signal proportional integral control connected in parallel. By this technique, a proportional-integral controller is called PI controller. If you use any certain standard PI control, input and disturbance input for the quick response and the steady-state condition can see that the error does not occur [8]. The following equation (3), the standard digital PI controller is the transfer function of controller.

$$
\mathrm{R}(\mathrm{z})=\mathrm{K}_{\mathrm{p}}+\frac{\mathrm{K}_{\mathrm{i}}}{1-\mathrm{z}^{-1}}
$$

In particular, the gain of the proportional controller $\left(\mathrm{K}_{\mathrm{p}}\right) \mathrm{P}$ gain in the steady state is reached quickly, while the steady-state error is made. The steady-state error has the effect to avoid that integral control gain $\left(\mathrm{K}_{\mathrm{i}}\right)$ I gain the steady-state error.

However, only one kind of the integral controller was tends to degrade the stability of the system Therefore, the integral control to the controller if you are using only the elements is extremely rare, and the control system to stabilize the steady-state error for constant input to avoid a proportional-integral controller was used. At this time, most of the PI controller, position control is resulting out the accumulation of integrator deviation called the Windup. When the controller is saturated with the controller that is longer transients, it is in response to a large overshoot and vibration. The smaller of the controller output limit output of the integrator becomes the larger and the longer the settling time of the response [9]. In order to prevent this Windup, Anti-Windup PI controller is used. The following block diagram of the Anti-Windup PI controller is equal to Figure 2.

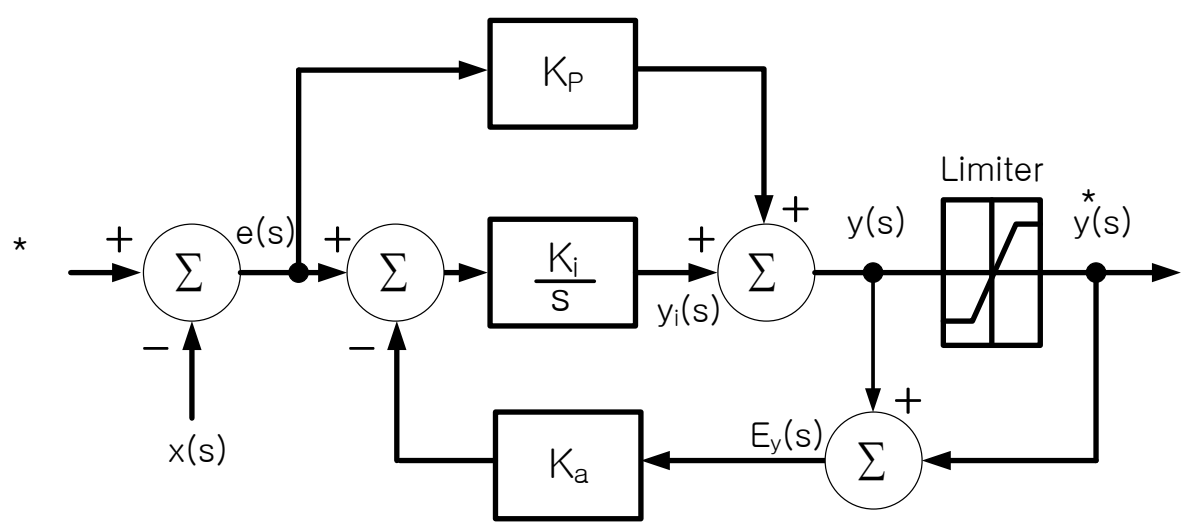

Figure 2. Anti-Windup Block Diagram

\section{Precise Attitude Control}

\subsection{Analysis of Gyro Sensor}

Mentioned earlier, the PI of the motor controlled the movement of the motor. The antenna can be easily controlled stopped state or when there is no disturbance to the correct one in the direction you want to, but if six degrees of freedom of movement, the antenna will have no way to respond to various disturbances. For this reason, it will require the gyro sensor. Gyro sensor have been used for attitude determination of the satellite and attitude control of the 
satellite body angular velocity to provide information to the most important sensors mounted on satellites. [10]

However, in recent years, the smartphone equipped with a gyro sensor is basically used in accordance with increasing penetration rate and the demand is rising in children. Gyro sensors mounted on rotating objects appears that the gyro precession produces a rotating repulsion, by measuring the force that is proportional to the value you add a device that generates an electrical signal to the gyro sensor is used. In x-axis, the equation of motion of the gyro sensor is equal to equation (4).

$$
\mathrm{F}_{\mathrm{x}}=\mathrm{mx}+\mathrm{c}_{\mathrm{x}} \mathrm{x}+\mathrm{k}_{\mathrm{x}} \mathrm{x}
$$

Constant m represents the mass of the structure, and $\mathrm{Cx}$ as the attenuation coefficient, $\mathrm{kx}$ as the spring constant, and Fx as the driving force of the electrostatic are equal to equation (5).

$$
\mathrm{F}_{\mathrm{x}}=\frac{\partial \mathrm{E}}{\partial \mathrm{X}}=\frac{\partial}{\partial \mathrm{X}}\left(\frac{1}{2} \mathrm{C}_{\mathrm{x}} \mathrm{v}_{\mathrm{D}}^{2}\right)
$$

$\mathrm{v}_{\mathrm{D}}$ Is one of the driving voltage, and the distance $\mathrm{x}$ is irrelevant. $\mathrm{Cx}$ is the capacitance between the beam and the resonant beam and it starts and switch the distance between the two and the capacitance values are changed. Thus, the capacitance $\mathrm{Cx}$ are as equation (6).

$$
\mathrm{C}_{\mathrm{x}}=\mathrm{C}_{0}+\Delta \mathrm{C}=\epsilon_{0} \frac{\mathrm{A}}{\mathrm{d}}=\epsilon_{0} \frac{\mathrm{t}}{\mathrm{d}}\left(\mathrm{x}_{0}+\Delta \mathrm{x}\right)
$$

In Equation (6), $\mathrm{t}$ is the thickness of com, and is the com overlap distance change. Thus, equation (5) is as equation (7).

$$
\mathrm{F}_{\mathrm{X}}=\frac{\partial \mathrm{E}}{\partial \mathrm{X}}=\frac{1}{2} \mathrm{v}_{\mathrm{D}}^{2} \frac{\partial \mathrm{C}_{\mathrm{x}}}{\vartheta \mathrm{X}}=\frac{1}{2} \epsilon_{0} \frac{\mathrm{t}}{\mathrm{d}} \mathrm{v}_{\mathrm{D}}^{2}
$$

Equation (7) can be seen in the fact that the driving force is proportional to the square of the driving voltage to the fact that the frequency of the resonant frequency of the driving voltage of $1 / 20000$ is the resonance even [11].

In addition, the translational motion gyroscope, regardless of the presence or absence of the element slope (angle) for the purpose to measure the change in the angular velocity and the angle measured from the reference point is calculated. Accelerometer to measure the acceleration of the speed and location information thrives, while a gyroscope to measure angular velocity and angular information is calculated. The angular velocity of the gyro sensor output (angular rate) that provides a direct form, increased incremental angle to provide forms and accumulated angle divided as to offer.

In this paper, we provide directly to the angular velocity of the gyro sensor, use the form. Gyro sensors posture and position estimation by using devices made for the inertial navigation system called inertial navigation system equipment. inertial system in motion (translational motion, rotational motion) detect and measure the distance and direction of the move and that the information will be output, and finally completely to track the movement in space, so this paper would like to applied to position control. 


\subsection{AGC Signal Detection and Initialization of Satellite Antenna Tracking Algorithm}

Existing commercial and residential portable satellite receivers, satellite center of the country with a specific target in the development and production of this family is. Rose of Sharon No. 3 in the case of the Republic of Korea broadcasts mainly residential (fixed) and automotive, marine products are a mainstay. However, depending on the country by continent plurality of goals, so there can be a single satellite to satellite tracking algorithms and tracking algorithms for the needs of the user by the target satellite cannot be changed. When the target is changed each time the satellite antenna and the control board that controls all of the algorithms FG worst to change can occur. In order to overcome these shortcomings of GPS information in real-time, i. e., NID (Network ID) to collect information that can be analyzed by applying the algorithm was able to solve the problem. When the check is complete, the NID Tuner Lock signal with a digital tuner AGC signal is generated. DBS receiver AGC signal is an indicator to determine the level.

Tuner Lock signal MCU internal interrupt is received, the $5 \mathrm{msec}$ level is checked every time the judge. Also, in a situation that does not move Tuner Lock signal ' 0 'is. However, the exercise of freedom corresponding to the movement of objects without system algorithms Tuner Lock signal is ' 1 'state is not received DBS. Tuner Lock signal is set to ' 1 'when the initialization process communication via satellite and can re-create the conditions are. However, many users in a time-consuming process to get the tuner to receive a lot of risk because no state will occur. The best Tuner Lock the device once the signal is not OFF precise attitude control is required. Mentioned above for precise attitude control, gyro sensor, and encoder, and the position of the tuner AGC control signal is implemented. Figure 4 indicates the tuner AGC output signal type.

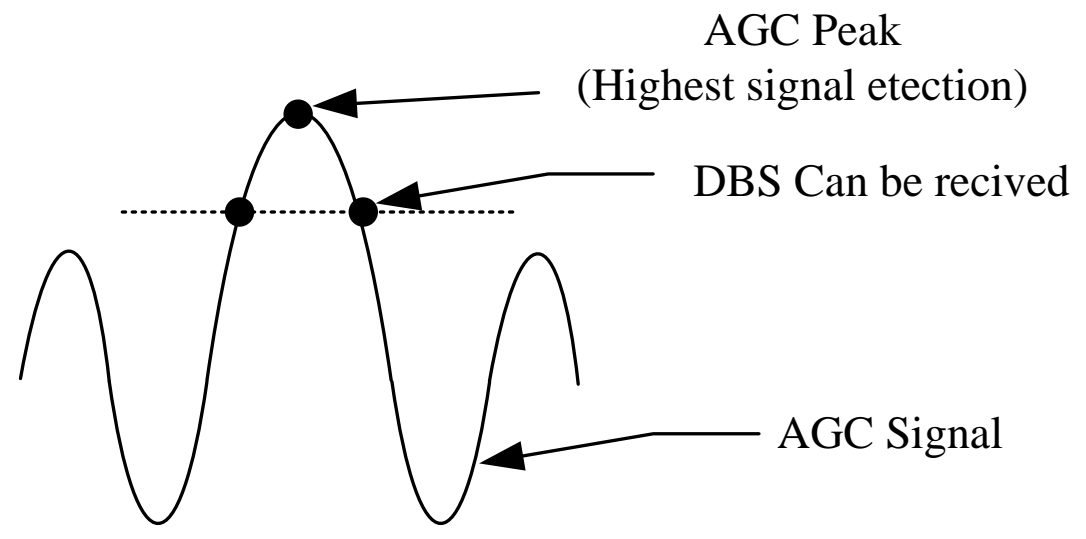

Figure 3. AGC Analog Output Voltage

AGC signal is an analog voltage output from the digital tuner that shows the signal strength of a satellite. AGC signal in the satellite antenna control is the most basic measurement values. Also compete with en-satellite and antenna azimuth axis control as is changing the angle of the AGC signal output value will vary accordingly. This motor is shown in Figure 3 AGC signal changes according to the movement, and when you reach the peak signal reception is the highest. AGC signal are required the high precision motor control algorithms in order to maintain the high reception. 


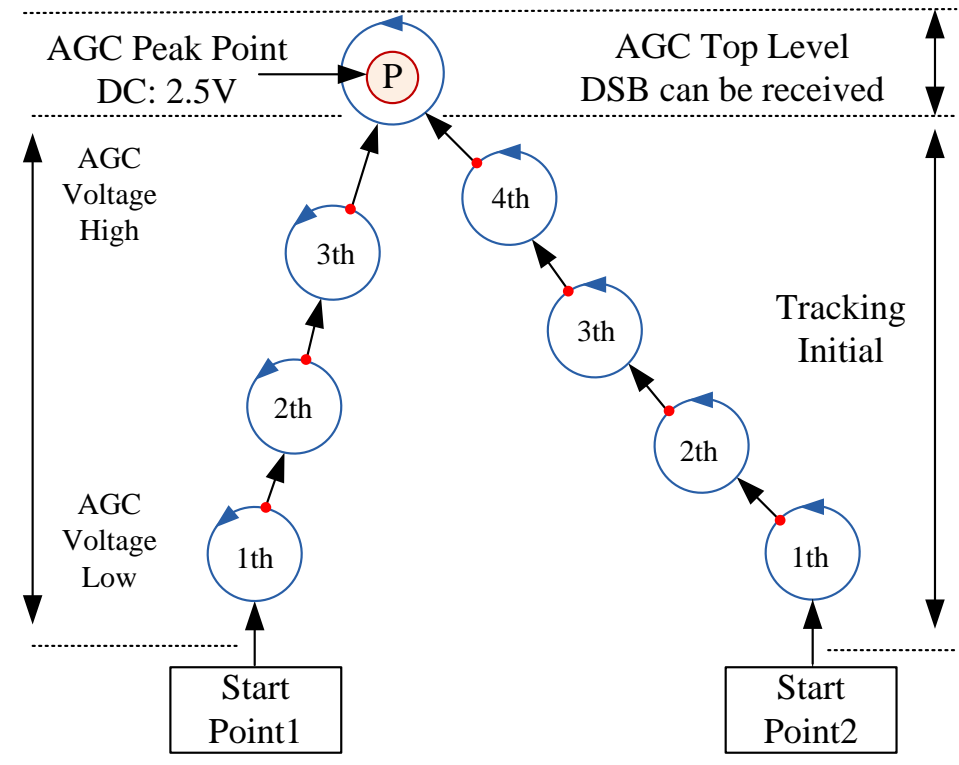

Figure 4. Block Diagram of the Circle Tracking Algorithm

System power is input to the AGC signal from the current position to the peak of the visiting process is shown in Figure 4. System initialization process, the first in the initial position of the antenna gyro sensor determines the initial position. It is 1th of Figure 4. While the antenna is rotated $360^{\circ}$ corresponding to the position of the AGC value to a digital value by the $\mathrm{AD}$ converter is stored in memory. After determining the initial position of the first lap, the antenna using the azimuth motor spins. In the second phase the antenna is moved in a circular motion AGC signal is detected. The AGC signal is detected based on the direction of the height of the antenna to move once again draw a circle to save the value of the AGC signal. So repeatedly in a circle will trace AGC signal. After tracking repeatedly 2th, 3th, 4th in Figure 5, when you reach the end of the peak point, based on Peak AGC signal points, the location of the antenna within $3^{\circ}$ making circle receive AGC signals periodically.

\subsection{2-Axis Bldc Motor Control Using Gyro Sensor}

In general, in the state still controlled two-axis motor position value if you have just the correct position, it is not difficult to control. However, in the context of six degrees of freedom movement from one place to gather around the disturbance must be done to overcome this first. In order to overcome the disturbances around the Yaw axis gyro sensor, Pitch axis, Roll all three axis gyro sensor output as an analog value to a digital value by the $\mathrm{AD}$ converter to convert the attitude control algorithms are applied. The location of existing motor control Yaw Pitch Axis axis calibration to finish, Roll-axis correction method was used to calibrate the general. In this paper, while Yaw, Pitch, Roll a position to compensate for the rotation axis, the three-axis direction at the same time, the algorithm was designed to follow. Figure 5 represents a change in the position according to the tracking of degrees of freedom, Yaw, Pitch axis and the axis movement. 


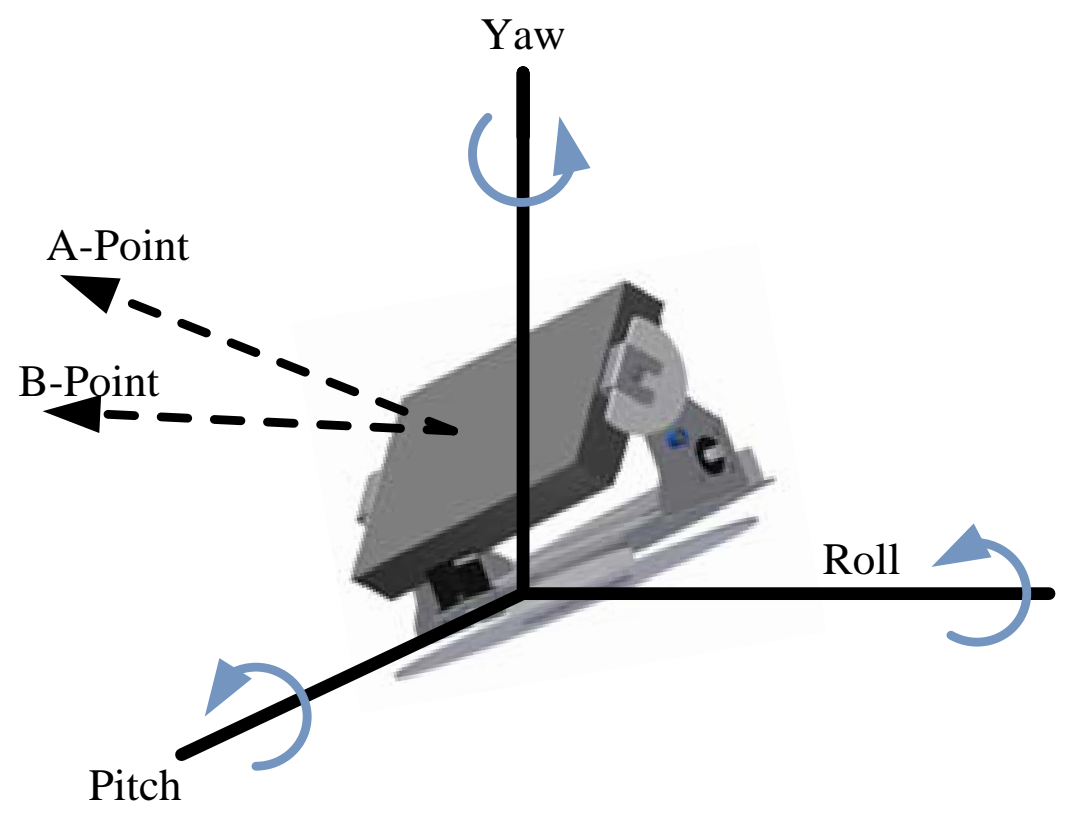

Figure 5. 6Degress of Freedom of the Antenna

In Roll-axis, rotating freedom of movement adjust the elevation to compensate the position, Pitch Yaw axis and the degrees of freedom of motion compensation axis azimuth is corrected by adjusting the position. In the design of the motor shaft and the axis Yaw Roll Pitch-axis 2-axis axis is designed to have no direct compensation. As a result, compared with 3-axis Enclosures physically tracking is insufficient. In order to overcome these shortcomings by attaching Pitch-axis gyro sensor tries to overcome the shortcomings. Directing point before moving in on the A-Point to B-Point directing point much difference due to the rotation axis Pitch Roll rotation axis by which the original signal tracking method is used.

Figure 6 is the order of the algorithm. The first proceed to initialize the MPU. Initialization part of the MPU internal AD converter available in the state, making the initialization of the gyro sensor, BLDC motors to control the initialization process to proceed. Enter the location tracking algorithm, the software won by an internal variable angle and the space that you want to enter an arbitrary angle. Track before the start of a three-axis gyro sensor attached to the analog value of the angular velocity data to the input of the AD converter receives. Gyroscope input for location tracking based on the go. Roll the basic location tracking algorithm based on the movement begins. As mentioned earlier, when you have moved en Roll different competing use of both the motor and the motor because the defense petitioned Roll is based on the algorithm was implemented. Awards and Awards Roll Yaw Pitch movement and en-compete when motion is detected on the motor and bearings inside the MPU competing motor PWM control is achieved through a two-axis control at the same time. PWM control and encoder value at the same time analyzing the movement distance and the angle of the motor can be calculated. Primarily to calibrate phase takes place in the time to just 5msec. Primarily in the state again, gyro sensor calibration data from the AD converter and then analyzed by the above method is executed. This can be control immediately in any disturbance of the above algorithm as well as it can follow the precise location. 


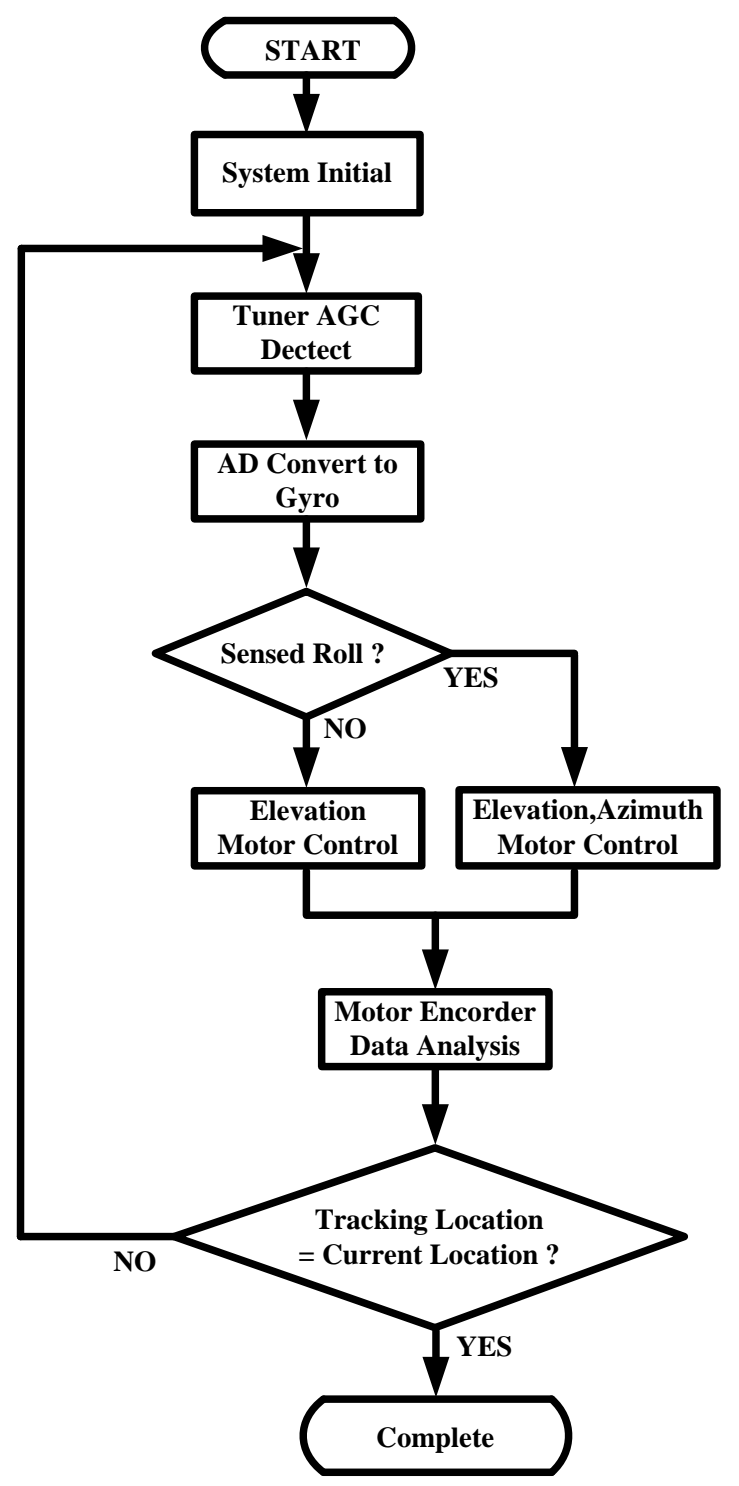

Figure 6. Software Algorithm for the Position Control of Motor

\section{Experimental Results and Discussion}

\subsection{System Construction and Design}

In this paper the basic specifications of the system and the system block is designed in equal to Figure 7. System on the part of the antenna design and the two-axis motor is installed, and Yaw, Pitch, Roll Gyro sensor is attached to the direction. Freescale's Cortex-M4-based control of the use MK60FX512VLQ12 MPU internal AD converter was used. The data communication between the MCU and the digital tuner was used for the I2C, AGC signal to an analog voltage value using the $\mathrm{AD}$ converter $\mathrm{MCU}$ internal data processing. Antennas and equipment were the direct design, the antenna and the associated satellite was connected to Sharon Koreaset3. 


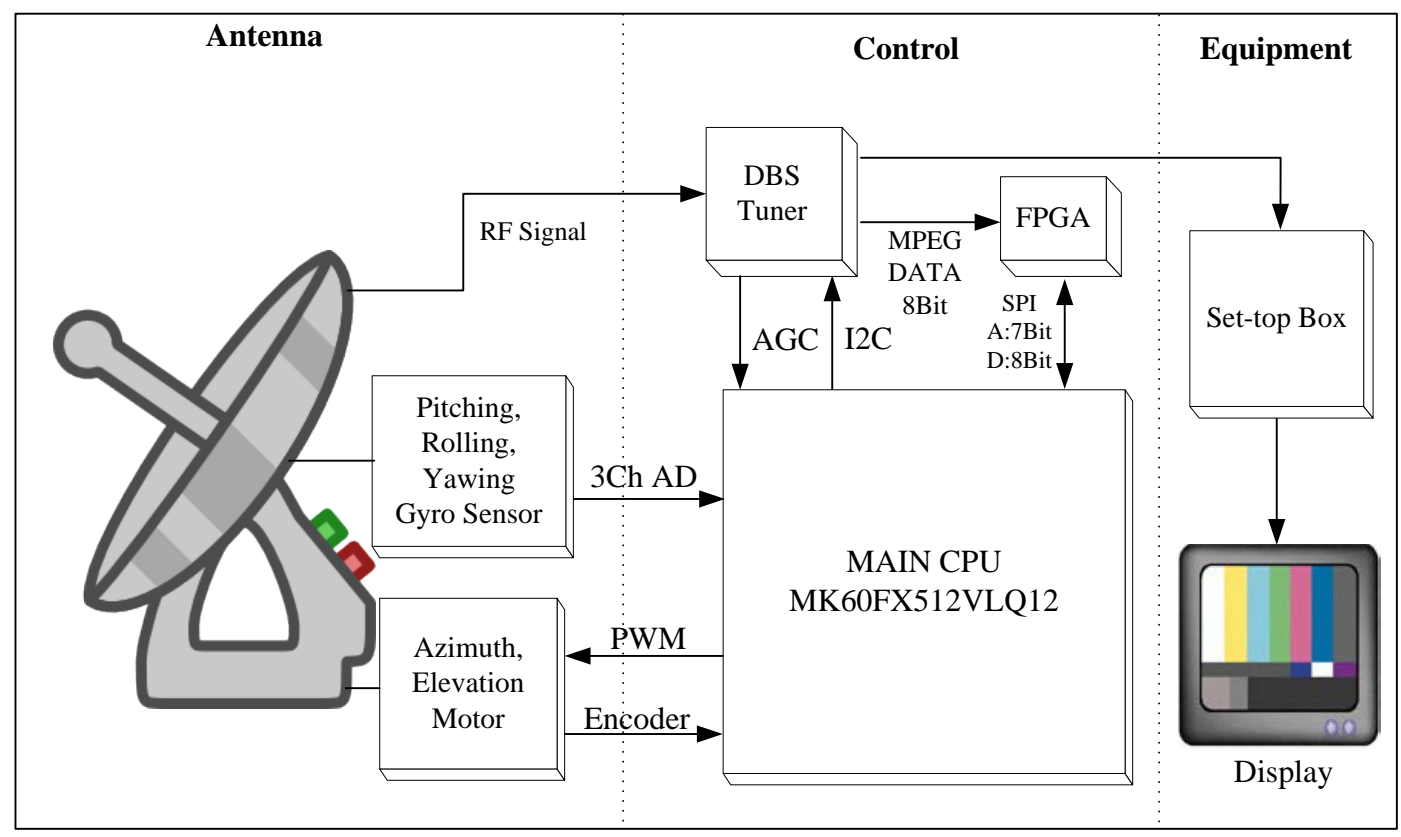

Figure 7. Block Diagram of the System

16Bit $\mathrm{AD}$ converter used the three channels to receive the analog value of a gyro sensor. Gyroscope using the XV-8000 series, and the motor was used for the BLDC motor, encoder 3150 pulses per revolution output, and the output pulse multiplied by 4 to 12,600 pulses per revolution was used. BLDC motor control used PWM module inside the MPU to control the motor, and the specifications of BLDC motor used in this paper shown in Table 2.

Table 2. Specification for the Motor

\begin{tabular}{cc}
\hline Parameter & Value \\
\hline \hline Number of poles & 4 \\
Rated Voltage(VDC) & 36 \\
Rated Speed(RPM) & 4300 \\
Rated Torque(N·m) & 0.22 \\
Rated Power(W) & 99 \\
Peak Torque(N·m) & 0.7 \\
Peak Current(A) & 11.5 \\
Line to line Resistance(ohms) & 0.64 \\
Line to line Inductance(mH) & 2 \\
\hline
\end{tabular}




\subsection{Experimental Disturbance Compensation}

6 degrees of freedom motion system designed to meet experimented. Figure 8 Gyro sensor output voltage waveform was measured using an oscilloscope.

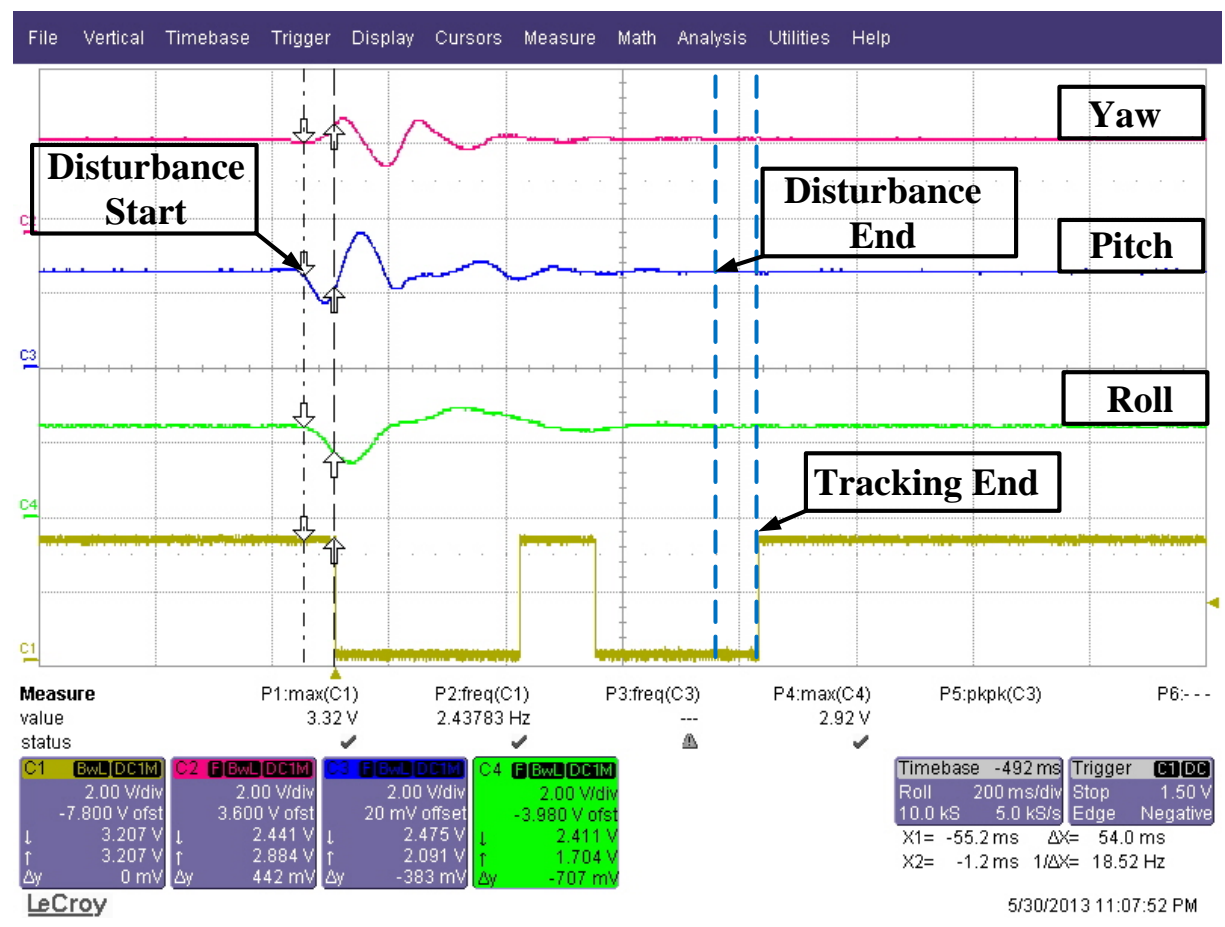

Figure 8. Disturbance of the Gyro Sensor and Motor Operation

In Figure 8 Yaw axis in the first line, second line is the Pitch-axis, and the third line is in the Roll axis gyro sensor output voltage. Figure 8 the status of the three axis antenna is a random disturbance authorized state. The fourth line displays the two-axis motor was time to move. High in the area of the antenna azimuth and elevation angle of $\pm 0.05^{\circ}$ in the position you want to keep believe that the motor is stopped, Low-in area to the antenna from the gyro sensor determines that disturbance will drive the motor. Yaw, Pitch, Roll-axis motion is detected in any one place, the motor response of about $30 \mathrm{msec}$ after a period of time was observed to be moving disturbance motor after $30 \mathrm{msec}$ after the stop at the same time tracking the movement is completed. After the disturbance, regardless of the disturbance at the end of the orientation angle of the antenna soon within $\pm 0.05^{\circ}$ return results were obtained. 


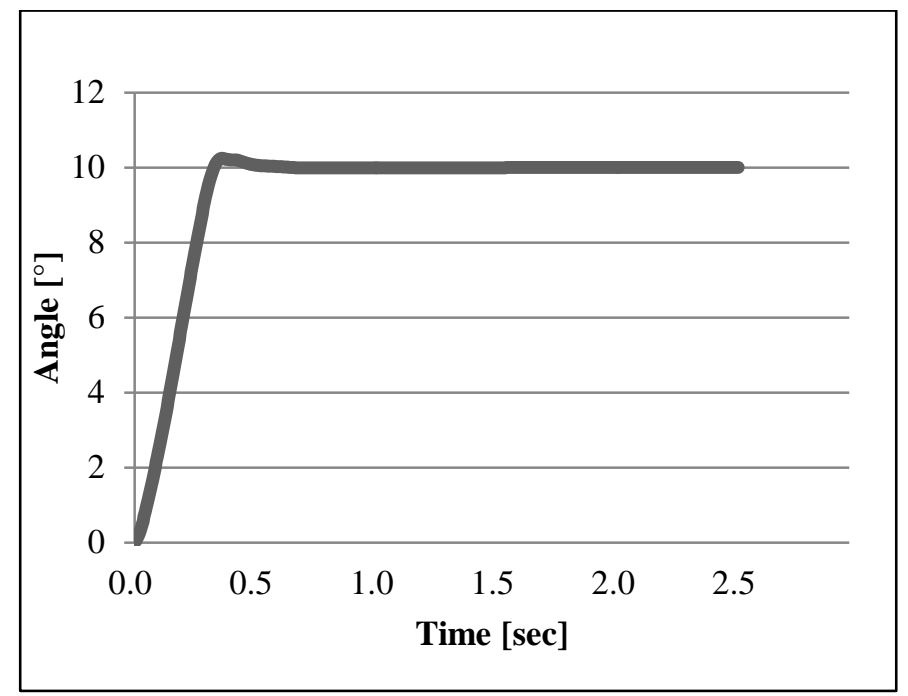

Figure 9. Step Input Response

In Figure 9 Target elevation of $10^{\circ}$ for the left track of the location is the result of one experiment. Antenna elevation angle from $0{ }^{\circ}$ to $10^{\circ}$ of the initial, enter Step Signal experimental time was 2.5 seconds were observed in approximately 0.4 seconds point overshoot of $0.24^{\circ}$ can be found to occur. This phenomenon appears when the disturbance of the antenna elevation and azimuth to control the gain of the PI controller, so this happens to be high. Enter the elevation of the antenna in the 0.6 seconds in the $10^{\circ}$ to $\pm 0.05^{\circ}$ angle of orientation of the antenna for the settlement was the result.
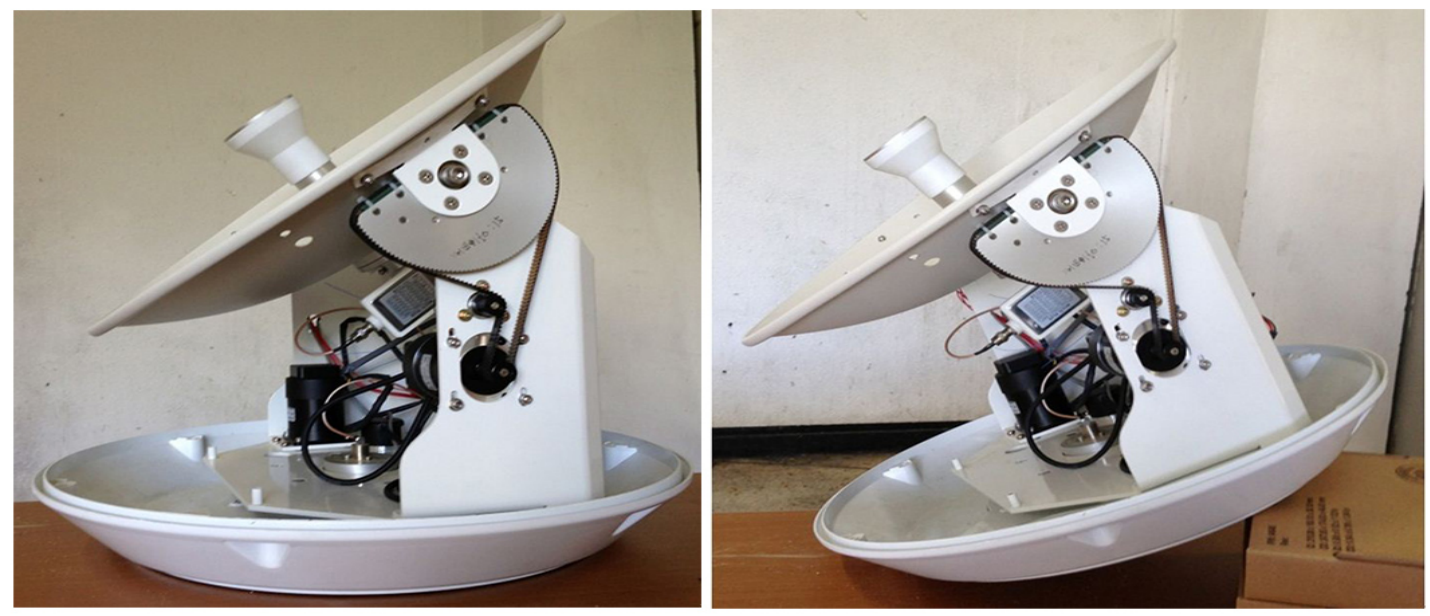

Figure 10. Fixed State for the Parabolic Antenna

In Figure 10 Right, the operating status of the prototype of the designed antenna is shown. Figure 10 is a fixed position in the state that holds the antenna picture. Orientation of the antenna elevation angle of $45^{\circ}$ can be seen that are moving. Figure 11 is when 6 degrees of freedom motion of the disturbance applied to the directivity of the antenna. It is applied to move as before disturbance. As shown in Figure 10, the orientation angle of the antenna to the external disturbance and was kept constant at $\pm 0.1^{\circ}$. 


\subsection{Experimental Satellite Tracking}

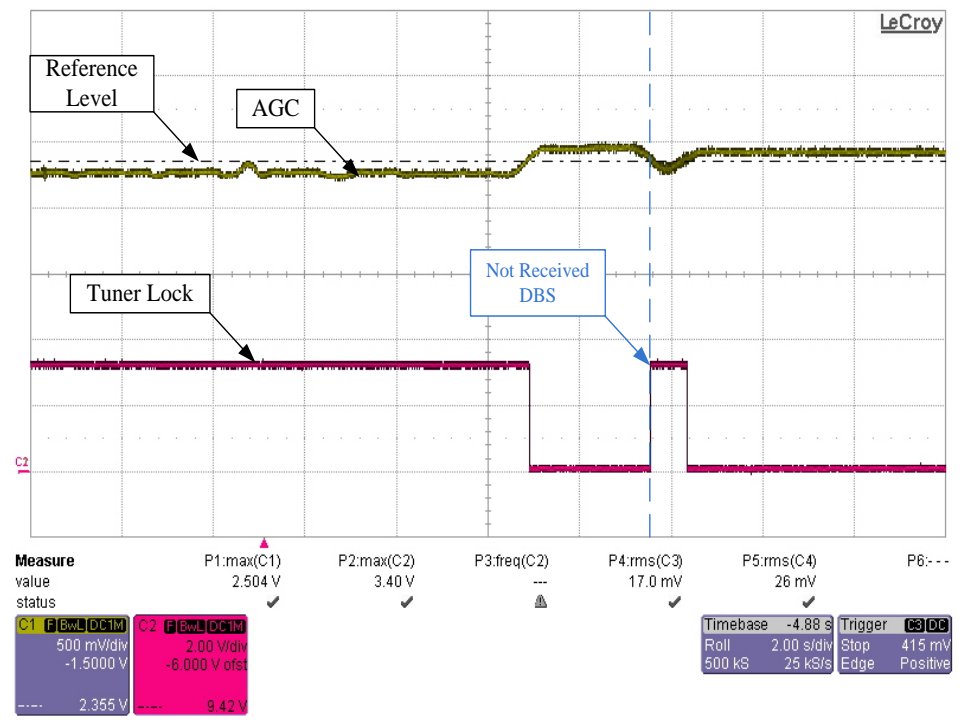

Figure 11. Antenna Start-up of the Previous Step Tracking Algorithm

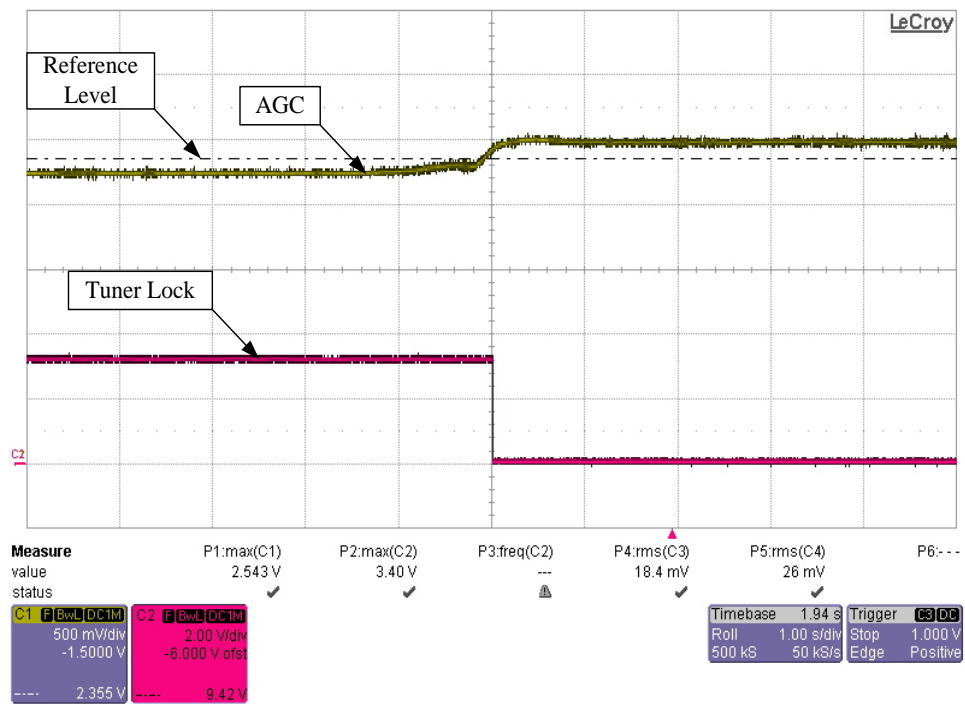

Figure 12. Antenna Start-up of the Proposed Tracking Algorithm

Figure 11 and Figure 12 are observed the waveforms of AGC in the initial tracking and Tuner Lock oscilloscope. DBS received signal availability Tuner Lock '0 'or, AGC Reference level should be higher than the signal can receive DBS. The tracking algorithm of Figure 11 is proposed in this paper and other Step Tracking Algorithm satellite tracking antenna system. Step-tracking algorithm to continue coming before the AGC signal and present the algorithm to compare the signal is coming. The issue of step-tracking algorithms DBS receiver AGC signal is that unstable. Figure 12 is waveform of presented tracking algorithm. Different from Step AGC tracking algorithm, we were able to confirm that the AGC signal has been maintained the level of the signal received DBS. 


\subsection{AGC Experiment when Disturbance Input}

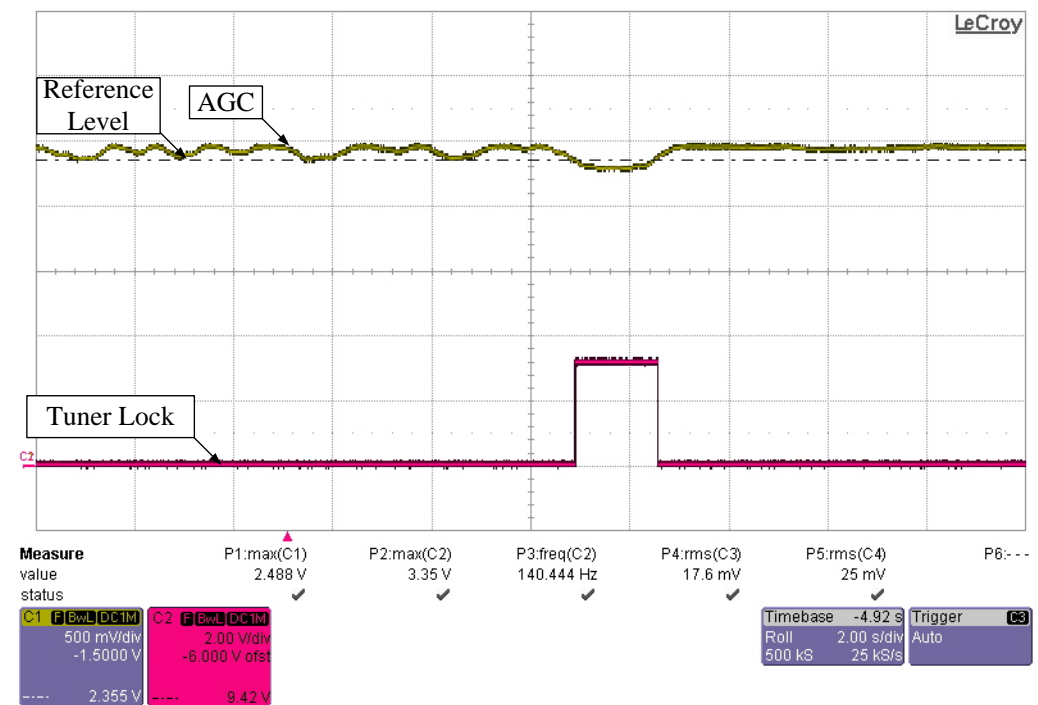

Figure 13. Disturbance Input Waveform of the Previous Step Tracking Algorithm

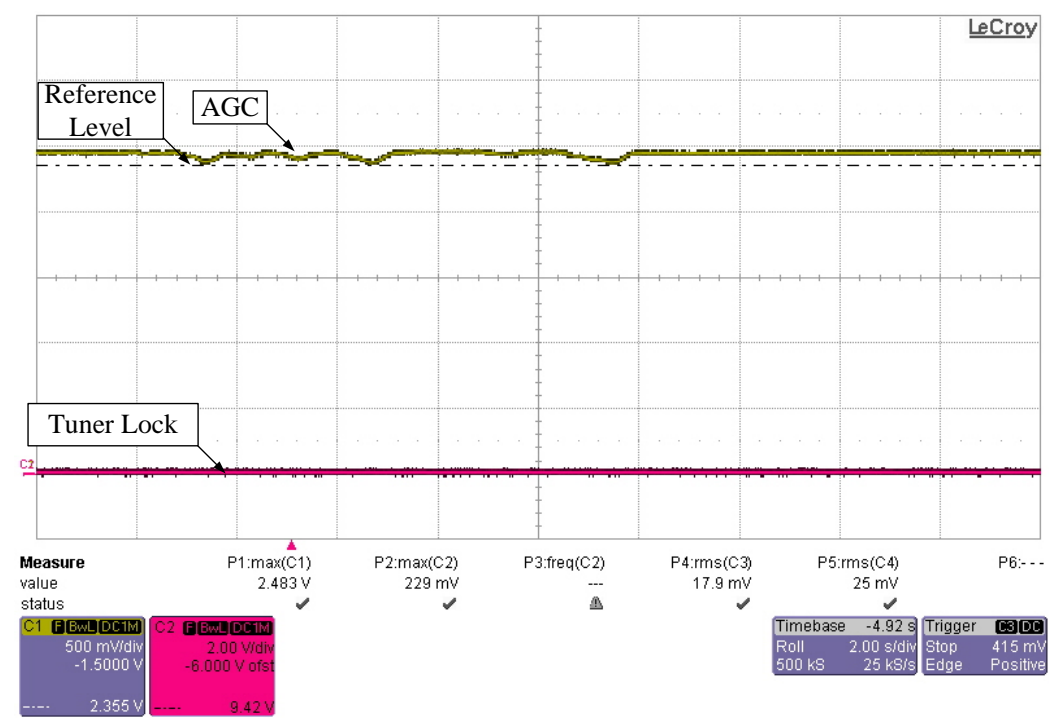

Figure 14. Disturbance Input Waveform of the Proposed Tracking Algorithm

Figure 13 and Figure 14 are the observed waveform of AGC in the time of the disturbance and Tuner Lock oscilloscope. Step-tracking algorithm of Figure 13, the disturbance is applied to the antenna at the Reference level AGC signal is smaller than the DBS choppy occur. However, the proposed algorithm applied waveform Figure 14 did not have such a phenomenon so we can see a AGC the signal Reference level to maintain. In this paper, we apply the proposed algorithm Step-tracking satellite tracking antenna system satellite tracking algorithm faster than the time of the initial tracking through the DBS receiver could be faster, but also to stabilize the AGC signal to the satellite can be received without interruption that were able to confirm. 


\section{Conclusion}

This paper aims to track the precise location of Parabolic satellite antenna using the BLDC motor, two-axis (elevation, azimuth), encoders, gyro sensor, digital tuner AGC signal and implements precise position control in the six degrees of freedom motion system. In order to overcome various disturbances gyro sensor Yaw, Pitch, Roll 3 axes were used. To respond immediately to the disturbance at the same time, two-axis motor by controlling the precise location of the system was designed to compensate instantaneously. Conventional stepping motor that was used for position control to the correct response, but not fast, but also a lot of noise caused by the shortcomings BLDC motor noise suppression, and also increase the reaction rate, the exact position encoder coupled high system was implemented to control the completeness. Digital tuner AGC signal between the antenna and the satellite can maintain high delivery ratio and orientation angle of the antenna to the desired angle by experiment that Step input to the desired angle within 0.6 seconds were able to confirm that. The disturbance of the antenna corresponding to the disturbance of the experiment, independent of the antenna orientation angle of the antenna soon recovered and an excellent location within \pm $0.1^{\circ}$ were able to confirm the control performance. The proposed Algorithm applied to satellite tracking antenna system is rather than Step-tracking, the initial tracking through satellite tracking DBS was able to receive faster, but also by stabilizing the AGC signal, it were able to confirm that the satellite can be received without interruption.

\section{Acknowledgements}

This research was financially supported by the Ministry of Education, science Technology (MEST) and National Research Foundation of Korea(NRF) through the Human Resource Training Project for Regional Innovation.

This work (Grants No. 2012-13) was supported by Business for Cooperative R\&D between Industry, Academy, and Research Institute funded Korea Small and Medium Business Administration in 2013.

\section{References}

[1] M. Won and S.-S. Kim, "Design and Control of a Marine Satellite Antenna”, Journal of Mechanical Science and Technology, vol. 19, no. 1, (2005), <Special Edition> pp. 473-480.

[2] J.-H. Oh, W.-Y. Koh, I. Jeong, S.-W. Hwang and W.-J. Shin, “A Study on the Design and Implementation of Stabilized Pedestal and Control Algorithm of Shipboard Satellite Antenna”, Trans. of the KICS, vol. 16, no. 3, (1997), pp. 451-455.

[3] J.-C. Choi and G.-S. Yang, "A Study on the DBS Receive Tracking Antenna Apparatus on a Ship by the Az/El Mount”, The journal of the Korea Institute of Maritime Information \& Communication Sciences, vol. 1, no. 2, (1997), pp. 209-220.

[4] New Technology company, 3-axial Acceleration Sensors (AM-3AXIS) Manual, New Technology Company.

[5] G. Yu and R. Hwang, "Optimal PID speed control of brush less DC motors using LQR approach", Proc. IEEE Int. Conf Systems, Man and Cybernetics, (2004), pp. 437-478.

[6] S.-K. Kim, B.-J. Han and H.-W. Yang, “The PSO-PID Speed Controller Design for the BLDC Motor”, The Transactions of the Korean Institute of Electrical Engineers, vol. 60, no. 9, (2011), pp. 1777-1782.

[7] S.-H. Kim, “DC, AC and BLDC Motor Control”, Bokdu publisher, (2010).

[8] S. K. Yoo and S. K. Jeong, "Precise Position Synchronous Control of Two-Axes System Using Two-Degreeof-Freedom PI Controller in BLDC Motor”, Journal of the Korean Society for Power System Engineering, vol. 5, no. 3, (2001), pp. 104-113.

[9] M. V. Kothare, "A Unified framework for the study of anti-windup designs”, Automatica, vol. 30, no. 12, (1994).

[10] I. S. Jung and K. H. Lee, “The advance of safety to inertia Sensor using at the Gyro Sensor”, KSME, vol. 37, no. 7, (1994), pp. 546-557. 
[11] Study on the micro gyroscope sensor IC design, National IT Industry Promotion Agency Academic articles, (1996).

\section{Authors}

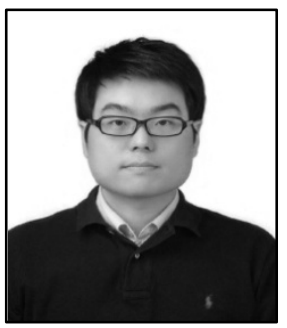

Myeongkyun Kim received his B.A. degree in Electronic Engineering from Cheongju University, Korea, in 2012. He is currently working toward the M.S. degree in Electronic Engineering from Cheongju University, Korea. His research interests are system design for industrial system and DSP control system

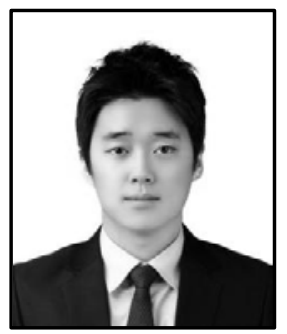

Jinsoo Kim received his B.A. degree in Semiconductor Engineering from Cheongju University, Korea, in 2013. He is currently working toward the M.S. degree in Electronic Engineering from Cheongju University, Korea. His research interests are satellite antenna tracking and DSP control system

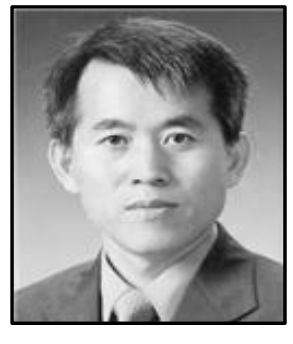

Oh Yang received his B.A., M.S., Ph.D. degree in Electrical Engineering from Han Yang University, Korea, in 1983, 1985, and 1997 respectively. From 1985 to 1997, he was research engineer at LGIS R\&D Lab. in the Department of Industrial Controller, An Yang, Korea. Since 1997, he has been with the Department of Electrical Engineering, Cheongju University, Korea. He was a visiting professor of A\&M University, Texas, USA, in 2006. He is currently a professor of the Semiconductor Engineering, Cheongju University, Korea. His research interests are converters for renewable energy, motor drives, power factor correction, and communication system for industrial controller. 